\title{
Dosing Observations and Pharmacokinetics by Surgery Type across Four Randomized, Placebo-controlled Trials with the Sufentanil Sublingual Tablet
}

Albert Dahan, MD, PhD1, Jean-Pierre Estebe, MD2, Maurice Jove, MD³

${ }^{1}$ Leiden University Medical Center, Leiden, Netherlands; ${ }^{2}$ Centre Hospitalier Universitaire Rennes, Rennes, France; ${ }^{3}$ DeKalb Medical Center, Decatur, GA

\section{Background}

Inpatient and outpatient postsurgical complications related to inadequate pain management negatively affect patient welfare and hospital performance. ${ }^{1-3}$

Poorly managed acute pain can lead to hypersensitization and within hours of onset, acute pain can provide physiological conditions that facilitate persistent pain syndrome or chronic pain.., 5 Multimodal approaches have demonstrated value but opioids still remain the cornerstone of acute post-operative pain management. ${ }^{6}$ The Sufentanil Sublingual Tablet System (SST 15 mcg; Zalviso $($ ), a PCA device dispensing $15 \mathrm{mcg}$ tablets, is approved in Europe for treatment of acute post-operative pain. A second sufentanil product, a 30 mcg tablet (SST $30 \mathrm{mcg}$ ) dispensed by a healthcare professional (HCP) in emergency medicine and short-stay surgery settings is also under review by the European Medicines Agency. Sublingual sufentanil has unique pharmacokinetic and pharmacodynamic properties and data evaluating two dosage formulations is now available across a broad range of orthopedic and abdominal surgery pain models. ${ }^{7-9}$ The primary objective of this post-hoc analysis was to evaluate drug utilization and sufentanil plasma concentrations by surgery type across the SST $15 \mathrm{mcg}$ and SST $30 \mathrm{mcg}$ pivotal trials.

Figure 1. Sufentanil Sublingual Tablet System and SST $30 \mathrm{mcg}$

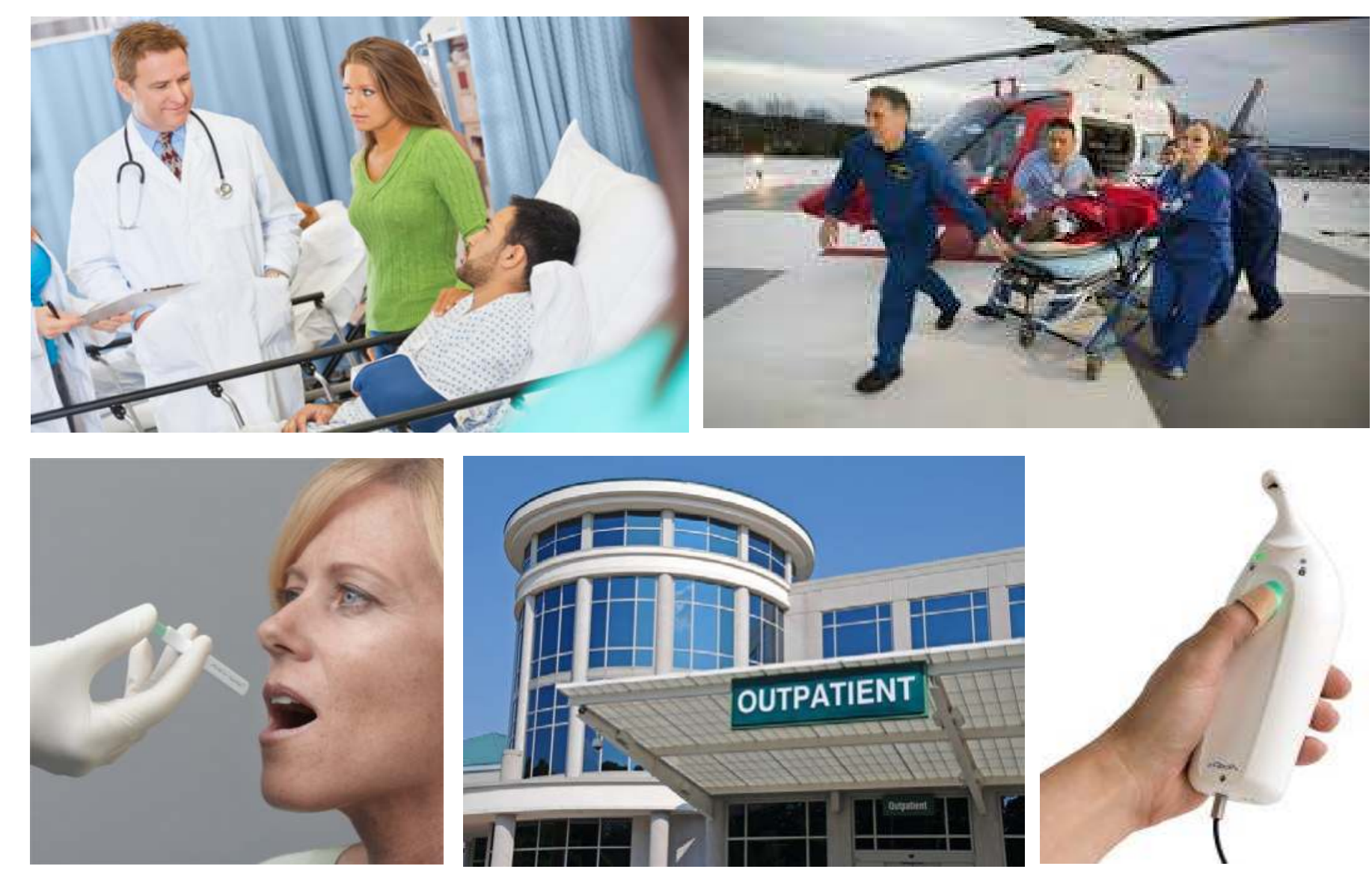

\section{Methods}

\section{Study Design}

- All studies were multicenter, randomized and placebo-controlled.

- Two were conducted with SST 15 mcg following open abdominal (ABD) surgery (IAP310) and knee (TKA) or hip (THA) replacement surgery (IAP311)

- SST 15 mcg patients were allowed to dose every 20 minutes as needed via PCA device

- Two studies were conducted with SST $30 \mathrm{mcg}$ following bunionectomy (SAP202) or abdominal surgery (ABD) including abdominoplasty,

laparoscopic abdominal surgery or hernia repair (SAP301)

- SST 30 mcg patients could receive a dose as needed throughout the study but not more frequently than hourly

- Before study staff could administer the first dose of study drug in any trial, patients must have reported a pain intensity (PI) score of 4 or higher on a validated, 11-point numerical rating scale (0-10).

- Rescue medication was available for all patients upon request

Efficacy Assessments

The primary efficacy variable in all studies was the time-weighted summed pain intensity difference (SPID) to baseline over the 48-hour (IAP310 and IAP311) or 12-hour (SAP202 and SAP301) study period

Key secondary endpoints included PI over the first hour, total pain relief (TOTPAR) and early termination due to inadequate analgesia

Detailed dosing data were collected and plasma sufentanil concentrations were derived from blood samples drawn at 12, 24 and/or 48-hours after the first dose.

Safety Assessments

- Safety assessments included adverse events (AEs), vital signs, including oxygen saturation, and the use of concomitant medications

\section{Results}

\begin{tabular}{|c|c|c|c|c|c|}
\hline \multirow{2}{*}{$\begin{array}{c}\text { Sufentanil } \\
\text { Dosing and } \\
\text { Plasma Drug } \\
\text { Levels (pg/mL) }\end{array}$} & \multicolumn{2}{|c|}{$\begin{array}{c}\text { IAP311 } \\
\text { SST } \\
\text { 15mcg }\end{array}$} & \multirow{2}{*}{ 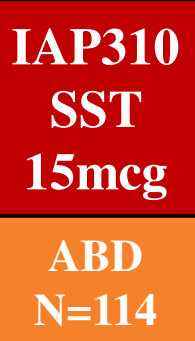 } & \multirow{2}{*}{$\begin{array}{c}\text { SAP202 } \\
\text { SST } \\
\text { 30mcg } \\
\text { BUN } \\
\text { N=40 }\end{array}$} & \multirow{2}{*}{$\begin{array}{c}\begin{array}{c}\text { SAP301 } \\
\text { SST } \\
\text { 30 mcg }\end{array} \\
\begin{array}{c}\text { ABD } \\
\text { N=107 }\end{array}\end{array}$} \\
\hline & $\begin{array}{l}\text { TKA } \\
\mathrm{N}=152 \\
\end{array}$ & $\begin{array}{l}\text { THA } \\
\mathrm{N}=163 \\
\end{array}$ & & & \\
\hline $\begin{array}{l}\text { Mean (median) } \\
\text { \# Doses, 0-12hrs } \\
\end{array}$ & $13(12)$ & $11(10)$ & $11(10)$ & $5(5)$ & $4(4)$ \\
\hline $\begin{array}{l}\text { Mean 12h Plasma } \\
\text { Concentration } \\
\end{array}$ & NA & NA & NA & 50 & 39 \\
\hline $\begin{array}{l}\text { Mean (med) } \\
\text { \# Doses, 0-24hrs }\end{array}$ & $23(22)$ & $21(20)$ & $20(18)$ & NA & 7(7) \\
\hline $\begin{array}{l}\text { Mean 24h Plasma } \\
\text { Concentration }\end{array}$ & 106 & 84 & 71 & NA & 44 \\
\hline $\begin{array}{l}\text { Mean (med) } \\
\text { \# Doses, 0-48hrs }\end{array}$ & $34(34)$ & $34(32)$ & $30(26)$ & NA & NA \\
\hline $\begin{array}{l}\text { Mean 48h Plasma } \\
\text { Concentration } \\
\end{array}$ & 97 & 85 & 69 & NA & NA \\
\hline
\end{tabular}

Baseline Demographics and Patient Disposition

- A total of 429 patients (152 TKA, 163 THA and 114 ABD) were randomized to SST $15 \mathrm{mcg}$; average age was 63 years, 64\% were female

- A total of 147 patients (40 BUN and $109 \mathrm{ABD}$ ) were randomized to SST 30 mcg; average age was 42 years, $63 \%$ were female

- Baseline pain intensity scores were lowest among hernia patients (5.0/10) and highest among abdominoplasty patients (6.5/10).

Efficacy

- Each of the four pivotal studies with SST met its primary endpoint of statistically superior pain reduction compared to placebo ( $\mathrm{p}<0.001$ for studies IAP310, IAP311, SAP301 and P=0.003 for SAP202)

- Dosing results indicate that patients undergoing orthopedic procedures (TKA and BUN) required more doses of SST to manage their postoperative pain than did patients undergoing ABD surgery

- Sufentanil plasma concentrations at all time points were higher among orthopedic vs abdominal surgery patients, with the SST 15 mcg PCA cohort titrating to comfort between $70-100 \mathrm{pg} / \mathrm{mL}$ vs $40-50 \mathrm{pg} / \mathrm{mL}$ for SST $30 \mathrm{mcg}$ patients that had shorter lengths of stay. Safety

- Table 2 includes AEs by surgery-type; nausea was the most common.

\section{Table 2. Common Adverse Events by Surgery Type}

\begin{tabular}{|l|c|c|c|c|c|}
\hline $\begin{array}{c}\text { Adverse } \\
\text { Event } \\
\%\end{array}$ & \multicolumn{2}{c|}{$\begin{array}{c}\text { IAP311 } \\
\text { \%ST 15mcg }\end{array}$} & $\begin{array}{c}\text { IAP310 } \\
\text { SST 15mcg }\end{array}$ & $\begin{array}{c}\text { SAP202 } \\
\text { SST 30mcg }\end{array}$ & $\begin{array}{c}\text { SAP301 } \\
\text { SST 30 mcg }\end{array}$ \\
\cline { 2 - 6 } & TKA & THA & $\begin{array}{c}\text { A BD } \\
\text { N=152 }\end{array}$ & $\begin{array}{c}\text { BUN } \\
\text { N=40 }\end{array}$ & $\begin{array}{c}\text { A BD } \\
\text { N=109 }\end{array}$ \\
\hline Nausea & 56 & 50 & 31 & 63 & 36 \\
\hline Pyrexia & 18 & 20 & 15 & 0 & 0 \\
\hline Anemia & 11 & 16 & 2 & 0 & 0 \\
\hline Vomiting & 11 & 15 & 9 & 28 & 9 \\
\hline Headache & 6 & 15 & 4 & 8 & 20 \\
\hline Pruritus & 7 & 9 & 9 & 10 & 3 \\
\hline O Sat Decr. & 5 & 11 & 6 & 0 & 1 \\
\hline Dizziness & 3 & 9 & 9 & 20 & 6 \\
\hline
\end{tabular}

\section{Conclusion}

Sublingual sufentanil was effective for the treatment of post-operative pain across a variety of short and long-stay surgeries Patients undergoing painful orthopaedic procedures such as TKA and bunionectomy may require more frequent dosing

Nausea was the most commonly reported AE across all studies

\section{References}

Jakobsson J. Pain Management in Ambulatory Surgery-A Review, Pharmaceuticals 2014,7:050 Meissson W. Pann Management in Ambulatory Surgery-A Review. Pharmaceuticals 2014:7:050-805 Awad and Ching Clin Exp Pharmacol Physiol Anesthesia and Pergolizzi JV, Jr., et al. Pain Manag Nurs. 2014; 15(1):380-9 Post-operative Pain Control Surg Clin North America; 2015;95: 301-318 ds. a pharmacologist's delight! Clin Exp Pharmacol Physiol 1995; 22:833-6. 2015):S90-S103. 